\title{
Numerical simulation of oscillations of a nonlinear mechanical system with a spring- loaded viscous friction damper
}

\author{
Viktor Nekhaev ${ }^{1}$, Viktor Nikolaev ${ }^{1, *}$, and Marina Safronova ${ }^{1}$ \\ ${ }^{1}$ Omsk State Transport University, 644046, Marx av., 35, Omsk, Russia
}

\begin{abstract}
The dynamics of a mechanical system consisting of a parallelconnected main elastic element, an external disturbance compensator having a nonlinear force characteristic, and a viscous friction damper sprung by a linear spring are studied. The resulting system of differential equations describing the behavior of the system has one and a half degrees of freedom and has specific properties depending on the ratio of stiffness of the main spring and the spring suspension of a viscous friction damper. It is established that a single nonlinear system with one and a half degrees of freedom has either one or two harmonics. In the general solution of the system of differential equations, there are always two harmonics in the above-resonance zone, one of which is always equal to the disturbance frequency, and the second one is sufficiently close to the frequency $\mathrm{k}_{0}$. In the linear conservative case and the absence of suspension of the viscous friction damper, the natural frequency of the displacement of the system $\mathrm{k}_{0}$ $=14.046 \mathrm{~s}^{-1}$.
\end{abstract}

\section{Introduction}

It is known that the introduction of an object into the standard vibration protection scheme consisting of an elastic element and an oscillation damper of the second disturbance transmission channel in which it is inverted, i.e. a compensating device (compensator), allows providing high parameters of its dynamic properties [1]. To protect the hydraulic damper from shock effects, an additional elastic element is used between it and the base. At the same time, the assessment of the influence of the stiffnessof an elastically suspended damper on the dynamics of such a system has not been fully studied.

\section{Methods of research}

Let's consider a mechanical system with a parallel installed main elastic element, an external disturbance compensator, and a spring-loaded viscous friction damper. The design scheme of the system is shown in Fig. 1, the nonlinear force characteristic of the compensator of the external disturbance is shown in Fig. 2. 


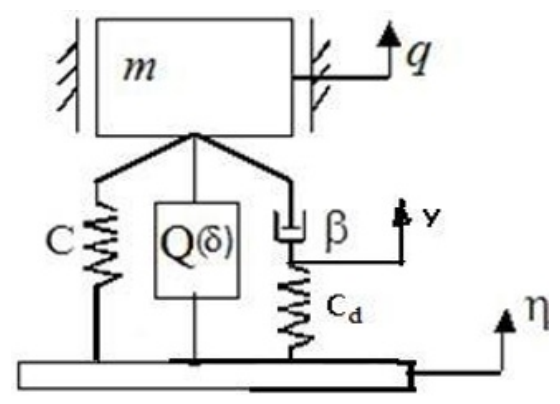

Fig. 1. The design scheme of a mechanical system with an external disturbance compensator and an elastically spring-loaded viscous friction dampener.

The following notations are introduced in the figure: $m$ - mass of the protected object, $C$ stiffness of the main elastic element, $C_{d}$-stiffness of spring connected in series with the viscous friction damper, $\beta$ - viscous friction coefficient of the system, $q$ - load displacement, $\eta$ - kinematic external disturbance, $Q(\delta)=a_{1} \delta+a_{3} \delta^{3}$ - power characteristic of the compensator of the external disturbance (Figure 2), $\delta=z-\eta$-deflection of the suspension.

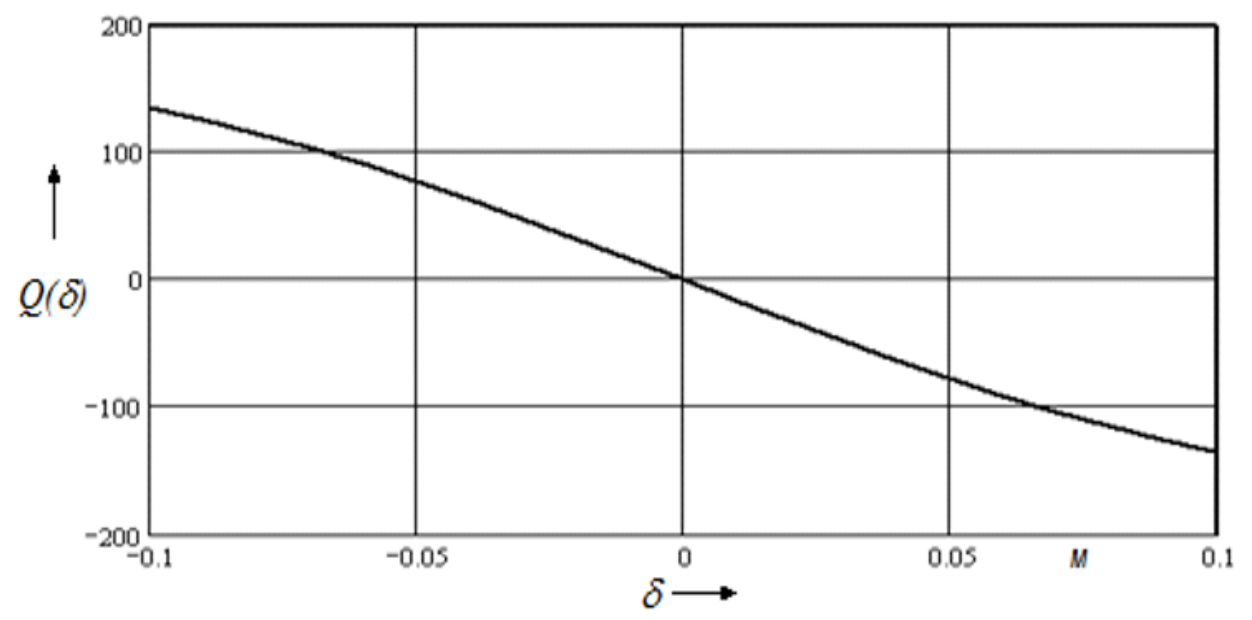

Fig. 2. Power characteristic of the compensator of external disturbance.

The design scheme of the mechanical system shown in Fig. 1 has one and a half degrees of freedom. Therefore, it is necessary to introduce one more generalized coordinate $y$ describing the displacement of the point of elastic suspension of the viscous friction damper. The dynamic behavior of the system is described by the following nonlinear differential equations:

$$
\left\{\begin{array}{l}
m \ddot{q}+\beta(\dot{q}-\dot{y})+C(q-\eta)+a_{1}(q-\eta)+a_{3}(q-\eta)^{3}=0 \\
-\beta(\dot{q}-\dot{y})+C_{\partial}(y-\eta)=0 .
\end{array}\right.
$$

In order not to integrate a system of differential equations with variable coefficients, we transform equations (1) to the form: 


$$
\left\{\begin{array}{l}
\ddot{\delta}+\gamma k_{0}^{2} y+k_{0}^{2} \delta+\frac{a_{1}+a_{3} \delta^{2}}{m} \delta=\gamma k_{0}^{2} \eta ; \\
-2 n \dot{\delta}+2 n \dot{y}+\gamma k_{0}^{2} y=-2 n \dot{\eta}+\gamma k_{0}^{2} \eta,
\end{array}\right.
$$

where $k_{0}=\sqrt{C / m}$ - natural frequency of a linear conservative system, $\gamma=C_{\partial} / C-$ ratio of the stiffness of the damper's springs and the main spring, $n=\beta / 2 m$-coefficient of damping of the system. We assume the harmonic function as a disturbance, taking into account that this mode of action on the vibrational mechanical system is the most unfavorable:

$$
\eta=h \sin \omega t,
$$

here $h$-amplitude of roughness, $\omega$ - frequency of external influence, $t$ - time.

To perform numerical integration of the system of differential equations (2), it must be represented in the normal Cauchy's form:

$$
\left\{\begin{array}{l}
\dot{x}_{1}=x_{2} ; \\
\dot{x}_{2}=\gamma k_{0}^{2} \eta(t)-\gamma k_{0}^{2} x_{3}-k_{0}^{2} x_{1}-\frac{Q\left(x_{1}\right)}{m} \\
\dot{x}_{3}=-\dot{\eta}(t)+\frac{\gamma k_{0}^{2}}{2 n} \eta(t)+x_{2}-\frac{\gamma k_{0}^{2}}{2 n} x_{3},
\end{array}\right.
$$

where $Q\left(x_{1}\right)=a_{1} x_{1}+a_{3} x_{1}^{3}$ - power characteristic of the compensator of external disturbance. This will allow us to find the phase relations between the excitation and the generalized coordinates.

\section{Results}

Fig. 3 shows the displacements of the load, the suspension point of the viscous friction damper, and the external disturbance.

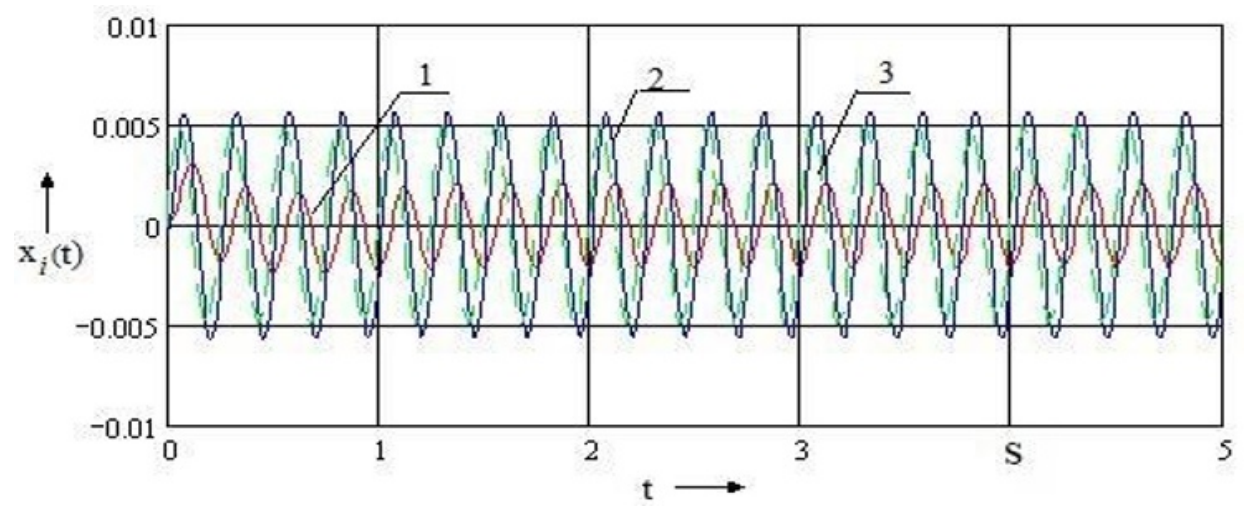

Fig. 3. Kinematic characteristics of a system with one and a half degrees of freedom, $\gamma=2: 1$ deflection of suspension $\left(x_{1}\right), 2$ - displacement of suspension point of viscous friction damper $\left(x_{3}\right)$, 4 - external disturbance $(\eta)$. 
From the above picture, the following fact is obvious: the angle of the phase shift between the displacements of the load exists, and to move the suspension point of the damper relative to the external disturbance, it can be considered equal to zero. This statement will allow us to carry out further analytical studies of the mechanical system under consideration, which is quite similar to the Duffing equation [2-6], which has jumping amplitude of oscillation. Consequently, something similar can be expected inthis mechanical system with one and a half degrees of freedom, but this phenomenon of jumps in the amplitude of oscillation is nearly impossible to reveal by numerical methods, but it is necessary to use the analytical method of solving the problem.

Fig. 4 presents a phase portrait of a mechanical system with one and a half degrees of freedom. At the end of the transient period of the process, periodic oscillations with amplitude of $0.6 \mathrm{~mm}$ and frequency equal to the external disturbance frequency of $4 \mathrm{~Hz}$ are established in the system.

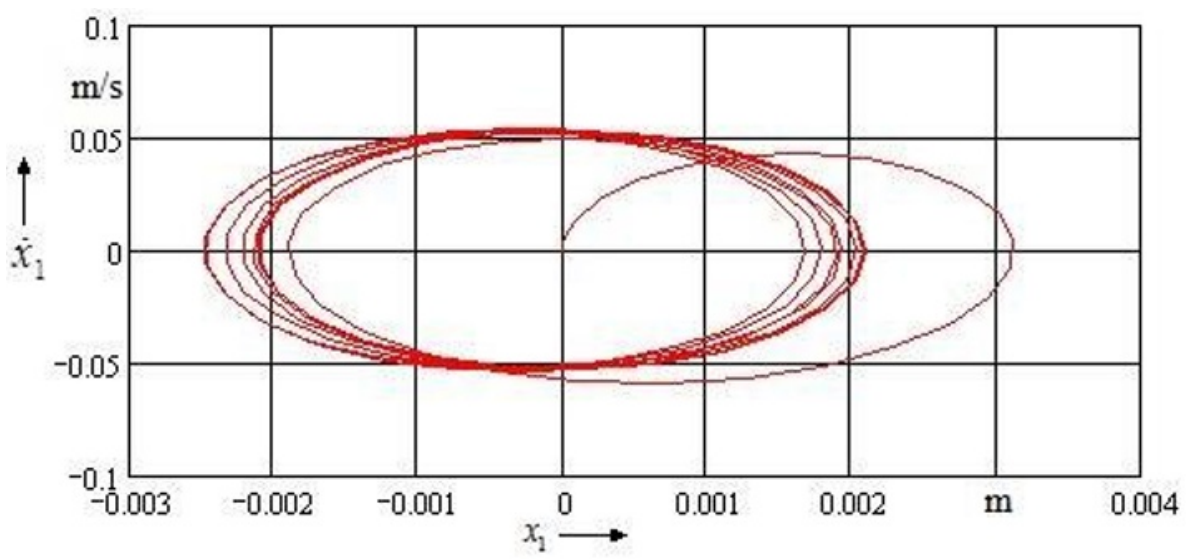

Fig. 4. Phase portrait of a mechanical system with one and a half degrees of freedom, $\gamma=2$.

The above results were obtained for the coefficient $\gamma=2$. If we reduce the coefficient $\gamma$ to 0,2 , i.e. ten times, the nature of the results will change significantly. For this case, the kinematic characteristics of a mechanical system with one and a half degrees of freedom are given in Fig. 5.

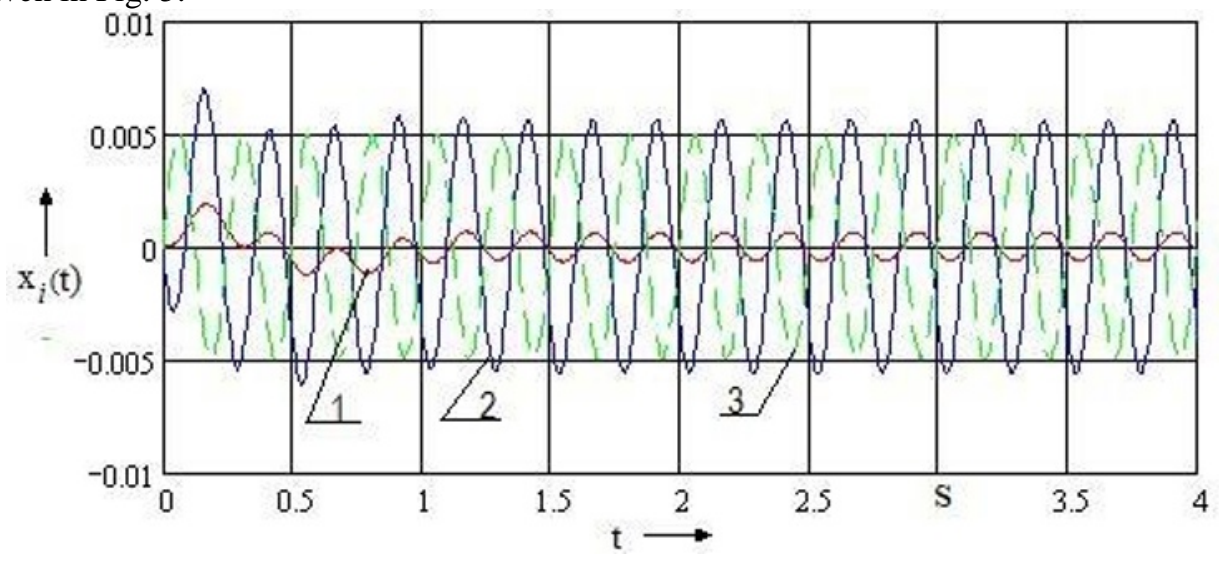

Fig. 5. Kinematic characteristics of a system with one and a half degrees of freedom, $\gamma=0,2: 1$ displacement of load, 2 - displacement of suspension point of viscous frictiondamper, 3 - external disturbance. 
As can be seen from this figure, the displacement of the load and the suspension point of the viscous friction damper occur synchronously, i.e. coincide in phase, and external disturbance with respect to them is shifted in phase. Fig. 6 shows the phase portrait of the dynamical system under consideration.

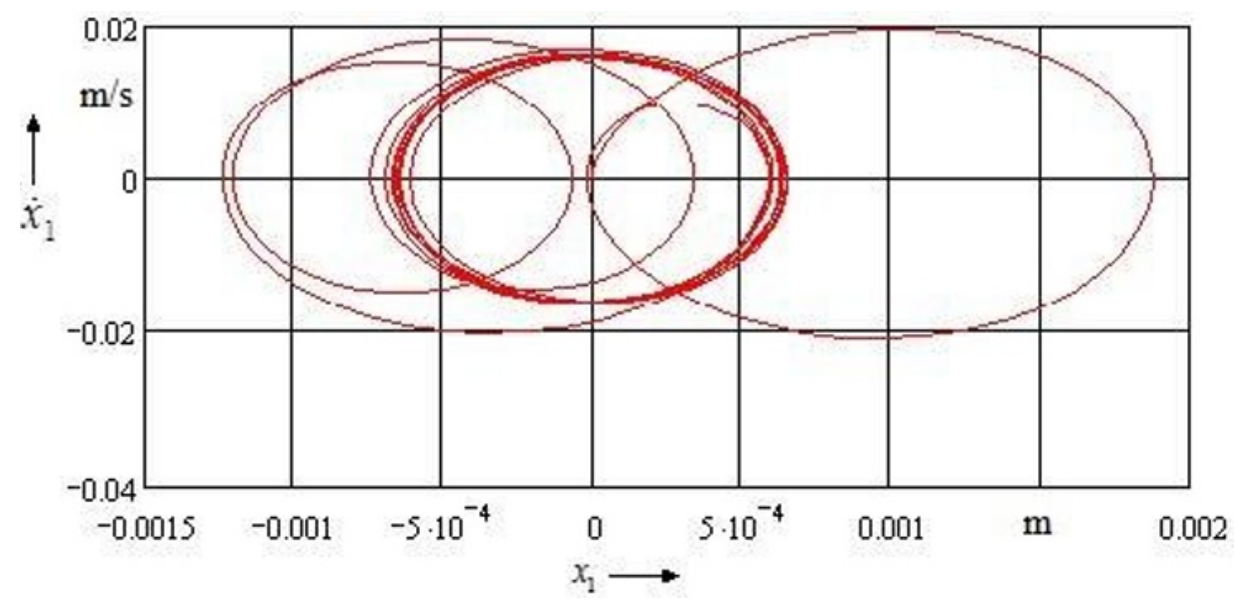

Fig. 6. Phase portrait of the dynamical system, $\gamma=0,2$.

The phase portraits shown in Fig. 4 (for $\gamma=2$ ) and Fig. 6 (for $\gamma=0.2$ ) substantially differ from each other. If in the first case the mechanical system "immediately" goes to the stationary regime, then in the second case it first goes to the right half-plane, then to the left half-plane, and eventually a stationary oscillation mode with an amplitude of $2 \mathrm{~mm}$ and the frequency of the external disturbing force is established.

So, the effect of the suspension of the viscous friction damper is the greater, the smaller the stiffness of spring connected in series with the absorber. Decreasing the coefficient $\gamma$ by a factor of ten, the amplitude of the displacement of load decreases by 3.335 times. But it is more important to note that for $\gamma=2$, the suspension point of the damper and external disturbance move synchronously, and for $\gamma=0.2$, the load and the suspension point of the damper move synchronously. This statement will be used in a further analytical study of the dynamic properties of a mechanical system with one and a half degrees of freedom.

It is well known [2 - 6] that harmonic components can be excited under certain conditions in nonlinear systems with frequencies both greater and lower than the disturbing frequency. To study this phenomenon, it is necessary to use spectral analysis [7, 8]. However, it should be recalled that the classical methods of calculating integrals, which are a tool of spectral analysis, are either the rectangle formula (this happens most often) or the trapezoid (this happens much less often). In this case, they cannot work efficiently because our random data contains latent periodicity. Therefore, based on the Philo's method [9, 10], an algorithm was created and a software package was written that was tested on test cases of random processes containing periodic components with an irrational frequency ratio. The program is implemented in the mathematical software package Mathcad of 13th version. The results of the spectral analysis of the oscillations of the system for various values of its parameters are presented below in Fig. 7 - 12. 
G,

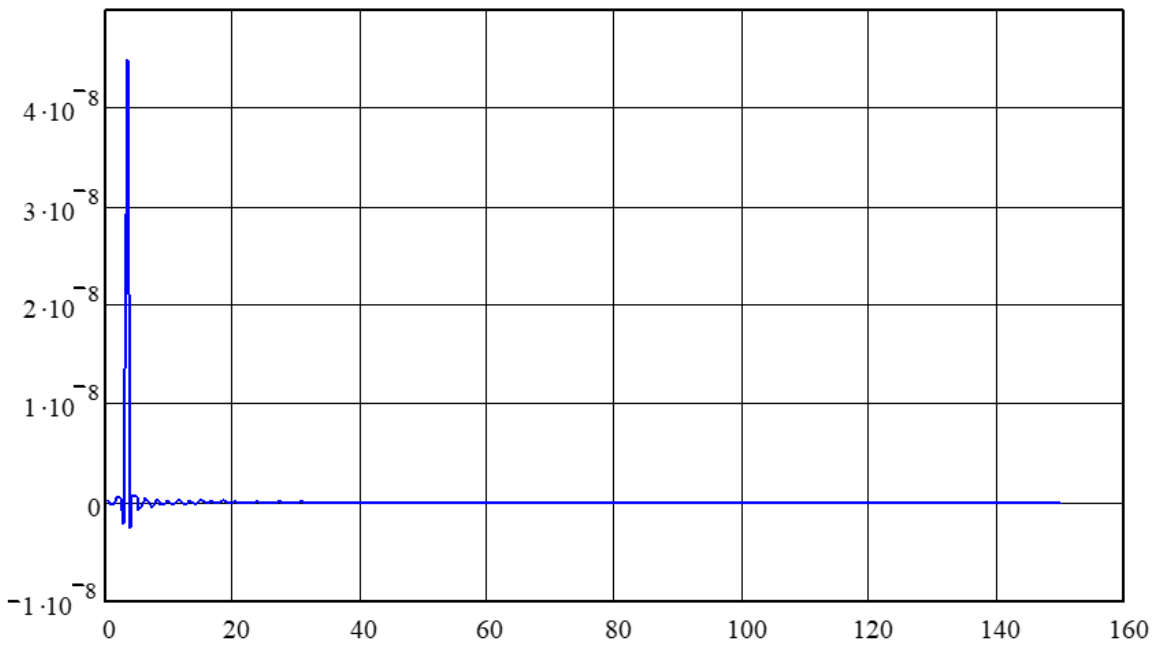

$\omega$,

$\lambda=0.25, \gamma=0,1$

a)

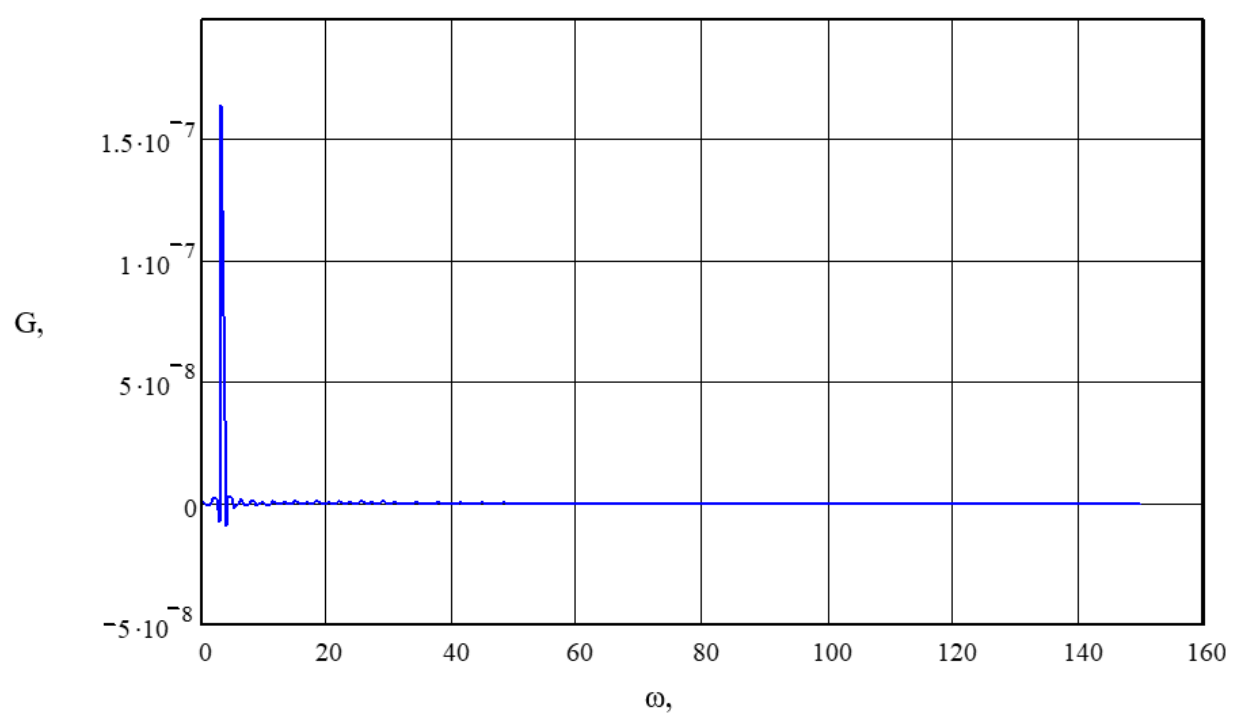

$$
\lambda=0.25, \gamma=0.4
$$

b)

Fig. 7. Spectral power of the displacement of the system, $\lambda=0.25: \gamma=0.1(a) ; \gamma=0.4(b)$ 


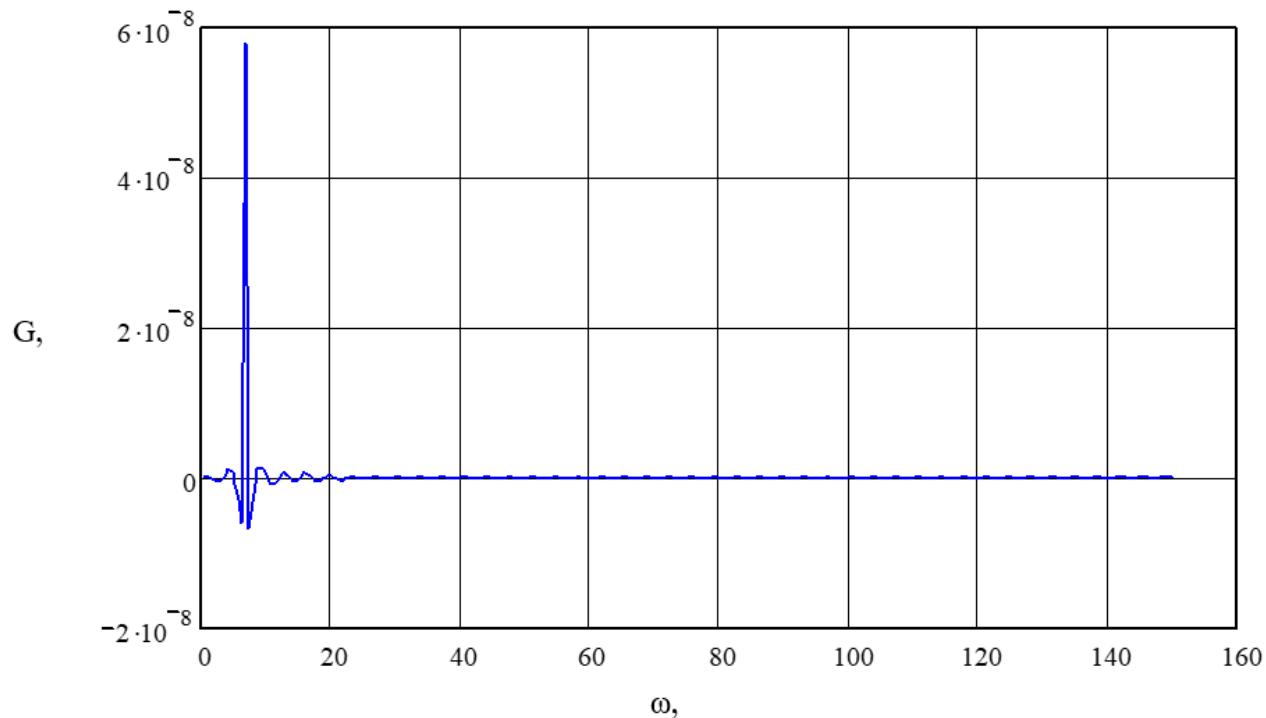

$\lambda=0.5, \gamma=0.1$

a)

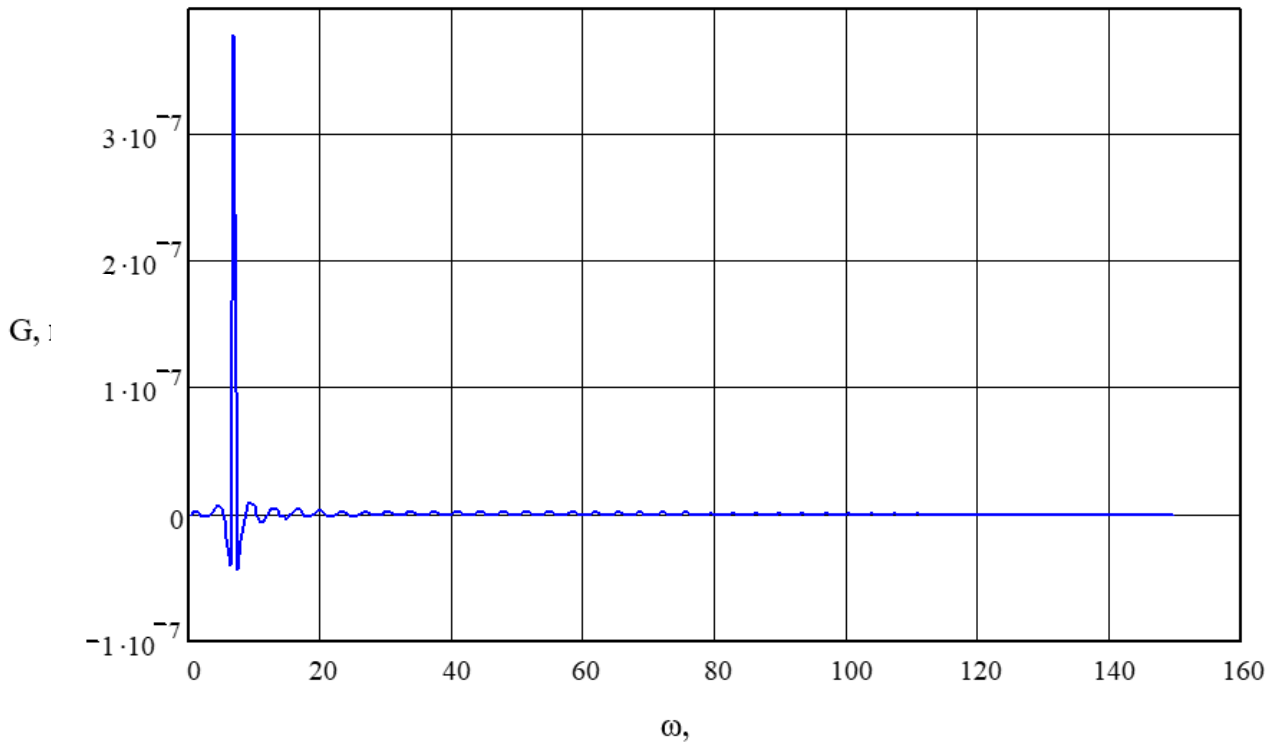

$\lambda=0.5, \gamma=0.4$

b)

Fig. 8. Spectral power of the displacement of the system, $\lambda=0.5: \gamma=0.1(a) ; \gamma=0.4(b)$ 


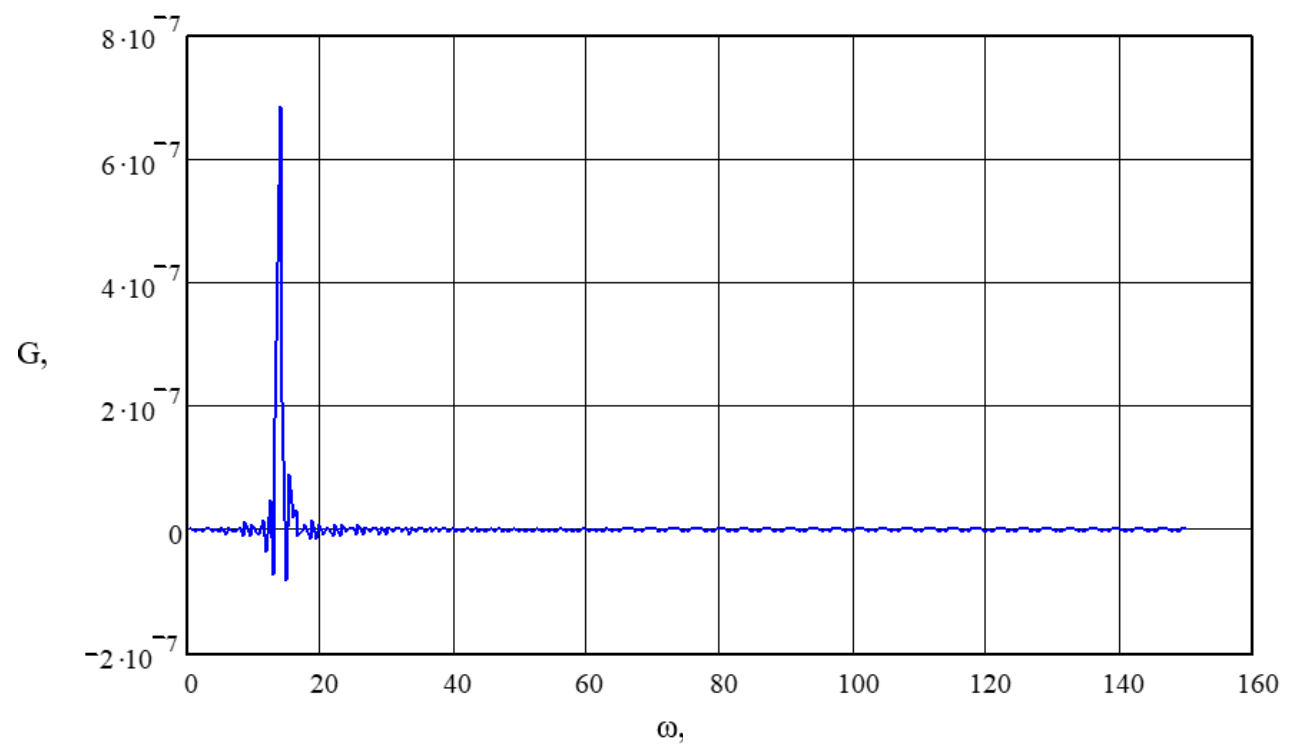

$$
\lambda=1, \gamma=0.1
$$

a)

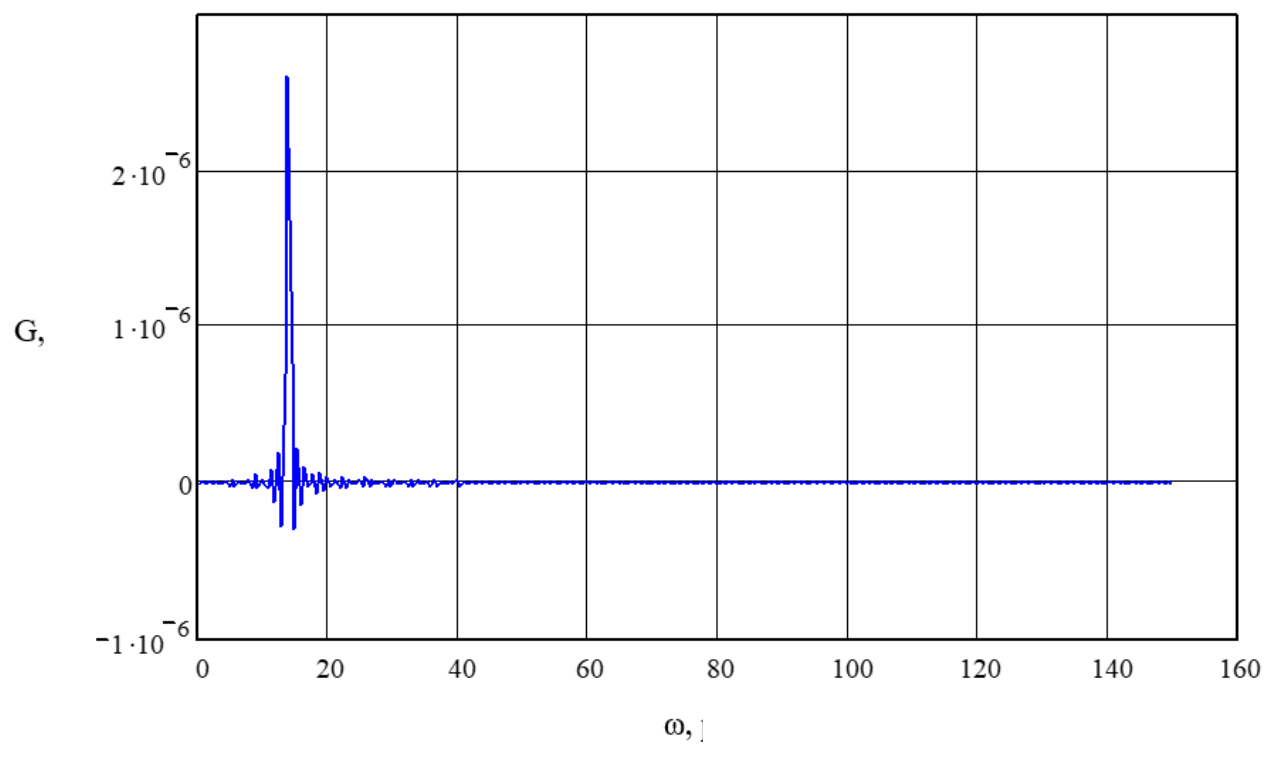

$$
\lambda=1, \gamma=0.4
$$

\section{b)}

Fig. 9. Spectral power of the displacement of the system, $\lambda=1: \gamma=0.1(a) ; \gamma=0.4(b)$ 
G,

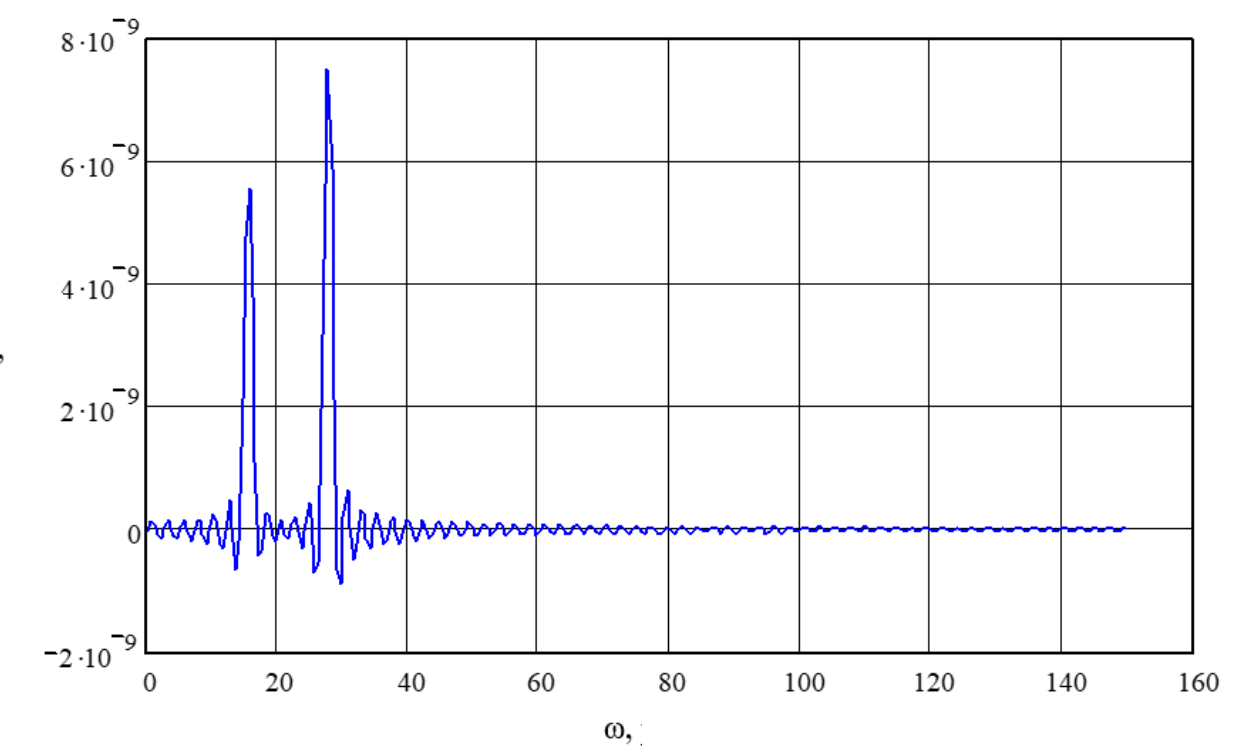

$$
\lambda=2, \gamma=0.1
$$

a)

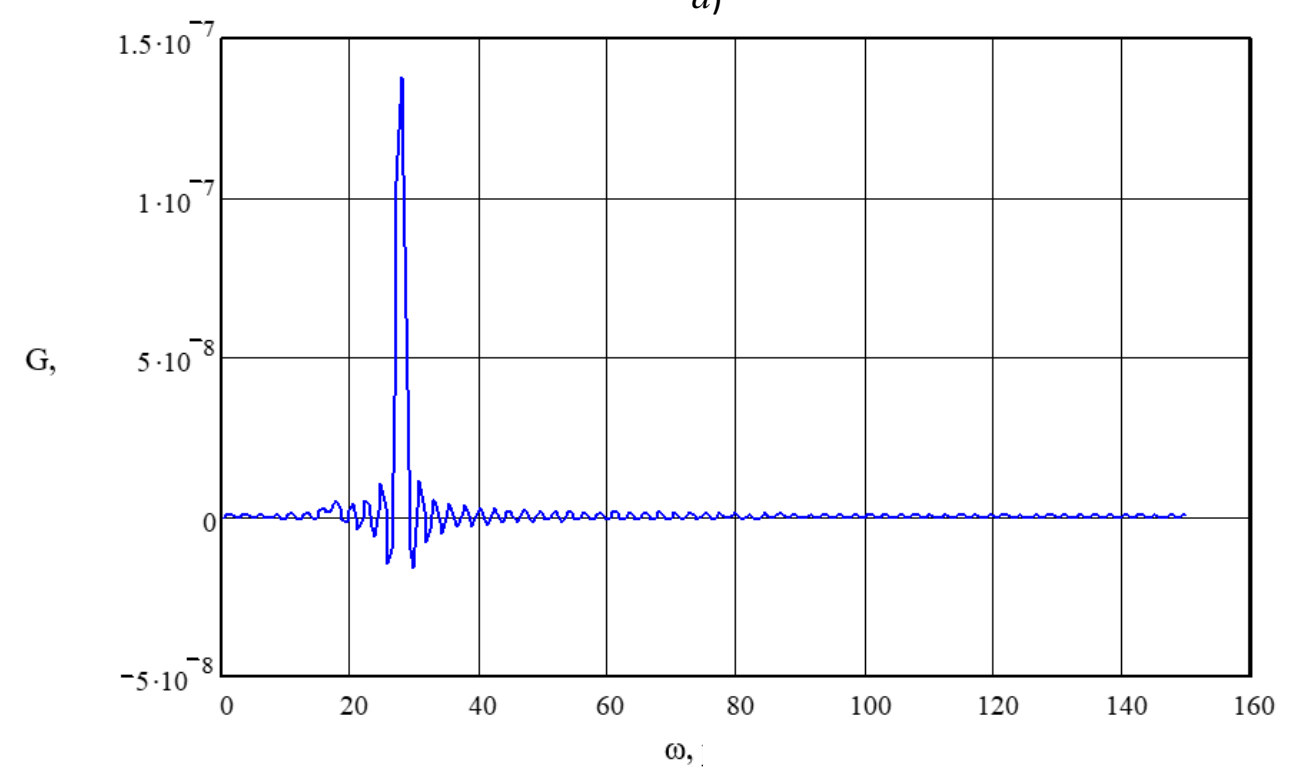

$$
\lambda=2, \gamma=0.4
$$

b)

Fig. 10. Spectral power of the displacement of the system, $\lambda=2: \gamma=0,1(a) ; \gamma=0,4(b)$ 


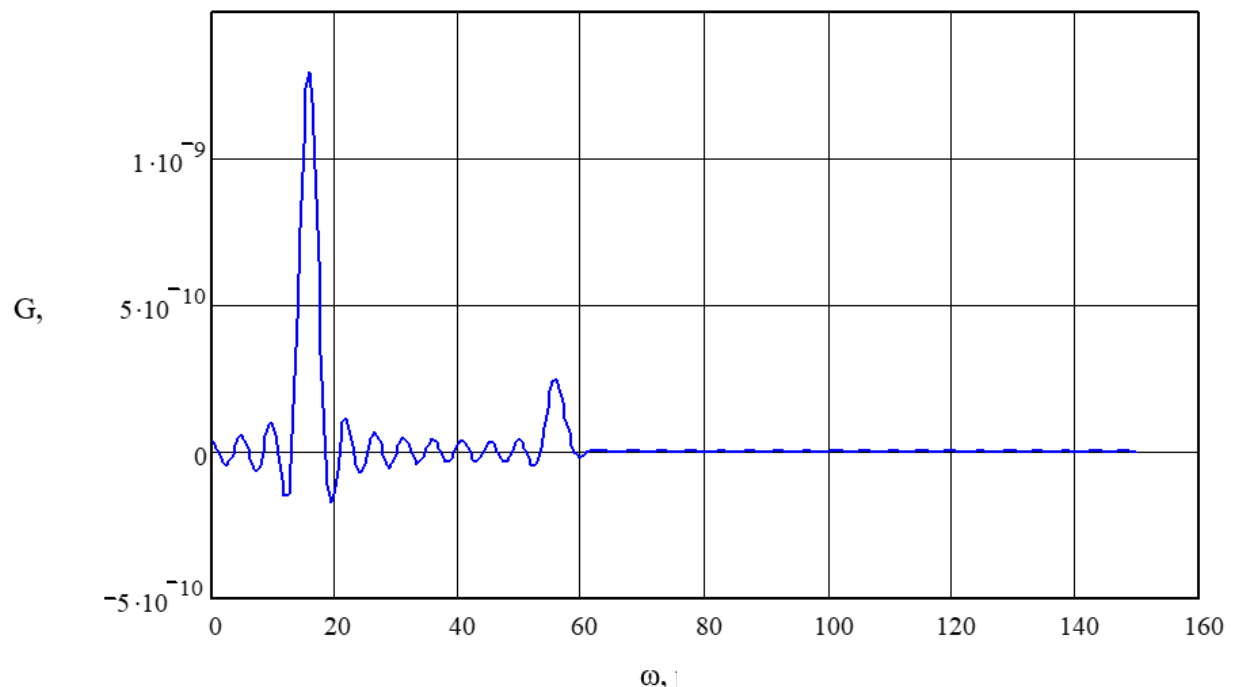

$\lambda=4, \gamma=0.1$

a)

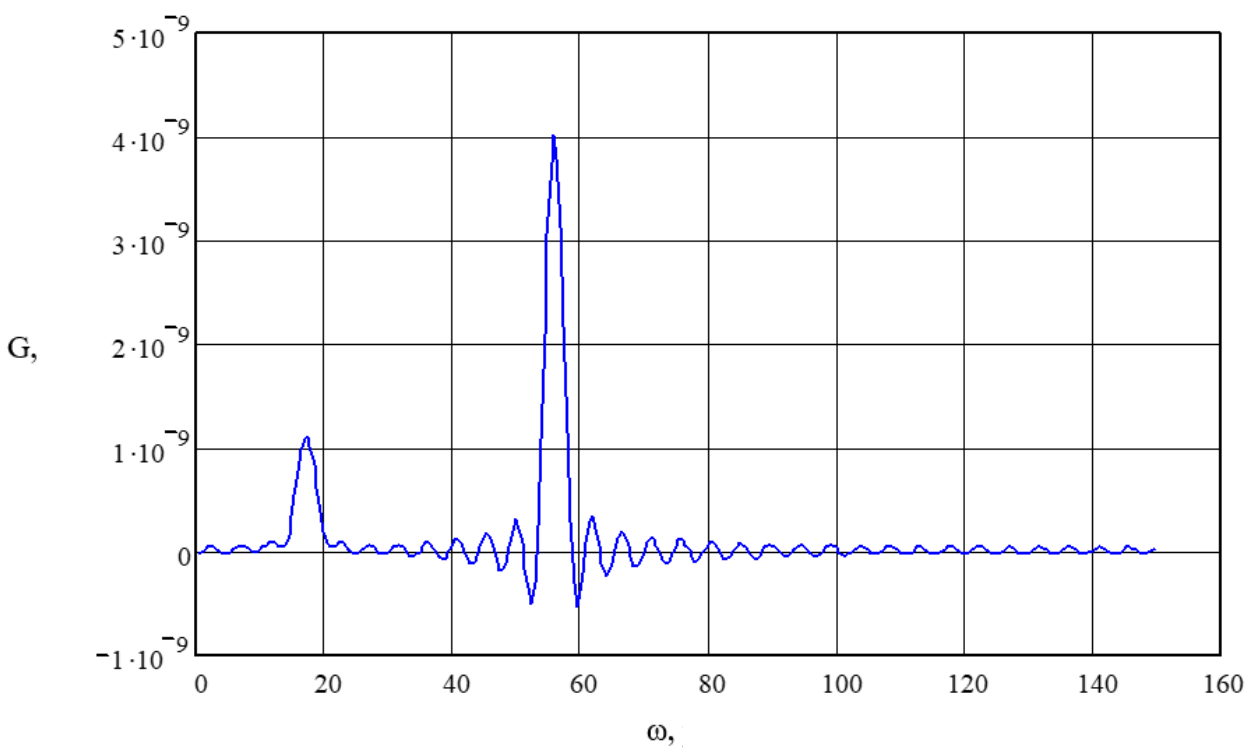

$$
\lambda=4, \gamma=0.4
$$

b)

Fig. 11. Spectral power of the displacement of the system, $\lambda=4: \gamma=0,1(a) ; \gamma=0.4(b)$ 


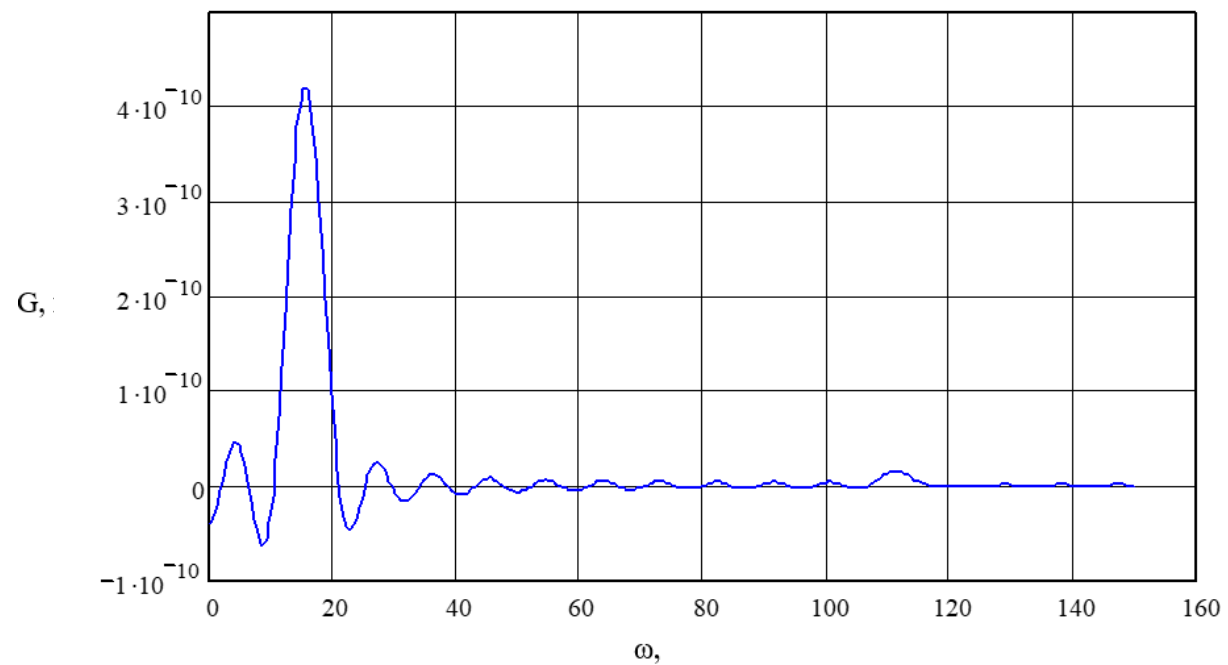

$\lambda=8, \gamma=0.1$

a)

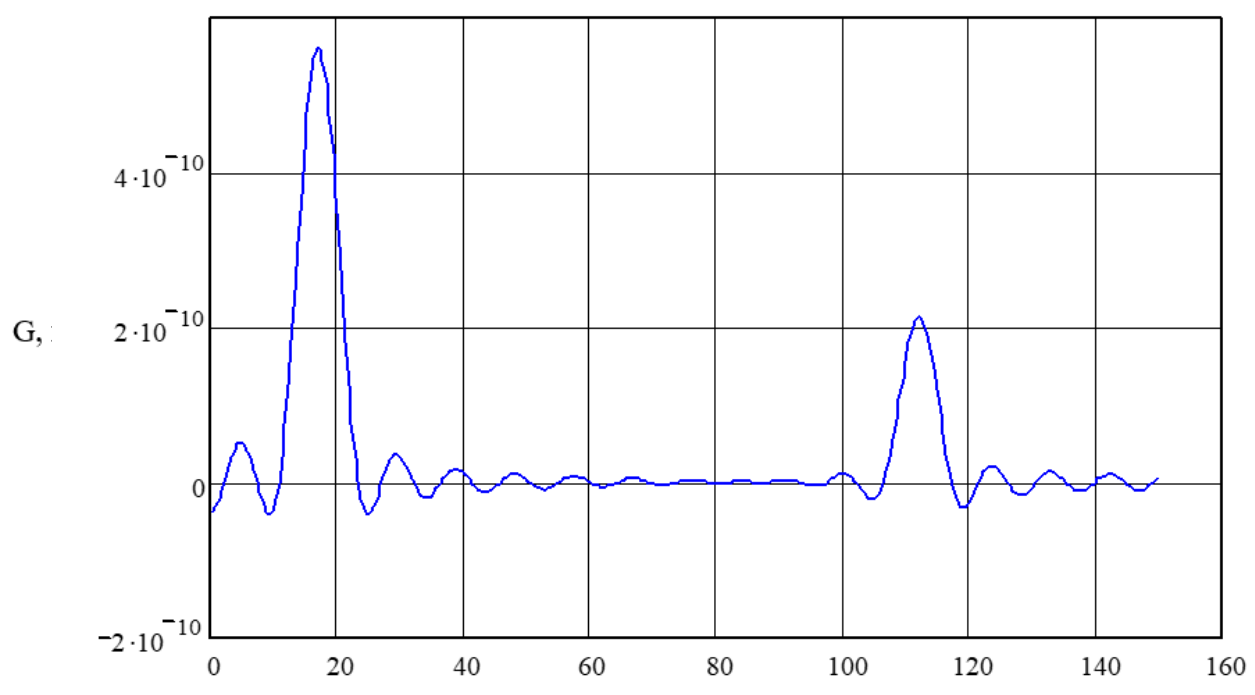

$\omega$,

$$
\lambda=8, \gamma=0.4
$$

b)

Fig. 12. Spectral power of the displacement of the system, $\lambda=8: \gamma=0,1(a) ; \gamma=0,4(b)$ 
From the graphs presented above, it is easy to conclude that in the considered nonlinear system with one and a half degrees of freedom, one or two harmonics are observed, which depends on the frequency detuning and on the ratio of the stiff nesses of the elastic elements. The frequencies obtained using the mathematical software package Mathcad are given in the table. Note that in the presence of a typical scheme of vibration isolation (parallel connected linear elastic element and unsprung viscous friction damper), the natural frequency of the system $\mathrm{k}_{0}=14,04642 \mathrm{~s}^{-1}$.

Table. Influence of the parameters $\lambda, \gamma$ and $\omega_{1}, \omega_{2}$ on the resonant frequencies of the dynamic system.

\begin{tabular}{|c|c|c|c|c|c|}
\hline No & $\begin{array}{l}\text { Frequency detuning } \\
\qquad \lambda=\omega / \mathrm{k}_{0}\end{array}$ & $\begin{array}{c}\text { Ratio of } \\
\text { stiffnesses } \gamma\end{array}$ & $\omega_{1}$ & $\omega_{2}$ & $\begin{array}{l}\text { Frequency of external } \\
\text { disturbance } \omega\end{array}$ \\
\hline 1 & \multirow[b]{2}{*}{0,25} & 0,1 & & 3,5 & \multirow[b]{2}{*}{3,5} \\
\hline 2 & & 0,4 & & 3,5 & \\
\hline 3 & \multirow[b]{2}{*}{0,5} & 0,1 & & 7 & \multirow[b]{2}{*}{7,02321} \\
\hline 4 & & 0,4 & & 7 & \\
\hline 5 & \multirow[b]{2}{*}{1} & 0,1 & & 14 & \multirow[b]{2}{*}{14,04642} \\
\hline 6 & & 0,4 & & 14 & \\
\hline 7 & \multirow[b]{2}{*}{2} & 0,1 & \multirow[b]{2}{*}{16} & 28 & \multirow[b]{2}{*}{28,09} \\
\hline 8 & & 0,4 & & 28 & \\
\hline 9 & \multirow[b]{2}{*}{4} & 0,1 & \multirow[b]{2}{*}{$16-17$} & 55,5 & \multirow[b]{2}{*}{56,019} \\
\hline 10 & & 0,4 & & 56 & \\
\hline 11 & \multirow[b]{2}{*}{8} & 0,1 & \multirow[b]{2}{*}{$16-17$} & 111,5 & \multirow[b]{2}{*}{112,04} \\
\hline 12 & & 0,4 & & 112,5 & \\
\hline
\end{tabular}

Analysis of the results of this table indicates the fact that in the general solution of the system of differential equations (2), there are always two harmonic sin the above-resonance zone, one of which is always equal to the disturbance frequency, and the second one is sufficiently close to the frequency $\mathrm{k}_{0}$. Of course, it is desirable to study the characteristic polynomial of the problem, but the system is nonlinear, so this is impracticable. It is also known that the natural frequency of a conservative nonlinear system varies with the amplitude of the oscillation.

It should also be noted that the concept of resonance for nonlinear systems is completely different than for a linear system where the coincidence of the frequency of external disturbance and the natural frequency of a conservative system is sufficient. In case of nonlinear systems, one should keep in mind not the ratio of frequencies but the ratio of work of the forcing and dissipative forces.

\section{The main conclusion of the paper is as follows}

As the spectral analysis of the results of numerical simulation of a nonlinear system with one and a half degrees of freedom showed, when the integration step was extremely small (the interval of integration was divided into 30,000 parts), the standard approach of the theory of oscillations would give a big error in estimating the displacement amplitude (see Fig. $12 a$ and $b$, from which it is obvious that the energy of the oscillatory process at a frequency $\omega_{1}$ is greater than at the disturbance frequency, while with an increase in the ratio of the stiffness $\gamma$, the oscillation energy at the disturbance frequency increases substantially). 


\section{References}

1. V.A. Nekhaev, V.A. Nikolaev, Synthesis of invariant vibroprotection system (theory and practice) Nonlinear vibration problems (DWH-Polish, Warszawa, 1993)

2. W. Cunningham, Introduction to the theory of nonlinear systems: translation from English(Gosenergoizdat, M.-L., 1962)

3. V.D. Goryachenko, Elements of the theory of oscillations (Vysshaya Shkola, Moscow,2001)

4. I.G. Malkin, Some problems in the theory of nonlinear oscillations (Gostekhizdat, Moscow, 1956)

5. M.Z. Kolovsky, Dynamics of machines (Mashinostroenie, L., 1989)

6. T. Hayashi, Forced oscillations in nonlinear systems (IL, Moscow,1957)

7. J. Bendat, A. Pirsol, Applied analysis of random data: translation from English (Mir, Moscow, 1989)

8. N. Draper, G. Smith, Applied regression analysis: translation from English (Finance and Statistics, Moscow, 1986)

9. A.F. Romanenko, G.A. Sergeev, Issues of applied analysis of random processes (Soviet Radio, Moscow, 1968)

10. V.A. Nekhaev, Interaction of vehicle with a quasi-invariant suspension system and a non-uniformly elastic track: dis.cand. tech. Sciences (1983) 